\title{
Breakage of sodium benzoate granules under repeated impact conditions
}

\author{
Ramanan Pitchumani $^{\mathrm{a}, *}$, Sarah Arce Strien ${ }^{\mathrm{b}}$, Gabriel M.H. Meesters ${ }^{\mathrm{a}, \mathrm{c}}$, \\ Stefan H. Schaafsma ${ }^{\mathrm{d}}$, Brian Scarlett ${ }^{\mathrm{e}}$ \\ ${ }^{a}$ Particle Technology Group, Department of Chemical Engineering, Delft University of Technology, Julianalaan 136, 2628 BL Delft, The Netherlands \\ ${ }^{\mathrm{b}}$ Research, Development and Technology, PURAC division, P.O. Box 21, 4200 AA Gorinchem, The Netherlands \\ ${ }^{\mathrm{c}}$ DSM Research, Food Specialties, P.O. Box 1, 2600 MA, Delft, The Netherlands \\ ${ }^{\mathrm{d}}$ DSM Research, Centre for Particle Technology, P.O. Box 18, 6160 MD, Geleen, The Netherlands \\ ${ }^{\mathrm{e}}$ Engineering Research Centre for Particle Technology, University of Florida, P.O. Box 116135, Gainesville, FL 32611, USA
}

\begin{abstract}
In many industries, handling or processing of relatively fragile particles takes place and predictions are required whether a significant proportion of the particles is damaged. Particles need to show good resistance against static and dynamic loads. The present paper deals with the study of particle resistance to dynamic loads (mainly the attrition behaviour) using a new instrument developed at the Delft University of Technology. The attrition test involves bombarding the particles against a flat target repeatedly, using a crank-slide mechanism. The new equipment generates extremely large number of collisions for each particle, resulting in reproducible generation of damage. The velocity is controlled by changing the frequency and amplitude of oscillation. The particles encounter many collisions on each spot on the particle surface during the test operation. The change in the particle size distribution at different intervals gives a measure of the damage caused to the particles. The damage has been characterised by estimating the specific rate of breakage, which is correlated to the frequency and amplitude of oscillation.
\end{abstract}

(C) 2004 Elsevier B.V. All rights reserved.

Keywords: Impact; Strength; Granules; Attrition

\section{Introduction}

It has been established that impact of particles causes attrition (Yuregir et al. [1]) where solution grown sodium chloride crystals of 1.2-mm cubes were accelerated in an air eductor and impacted onto a target plate at a velocity of 29 $\mathrm{m} \mathrm{s}^{-1}$. The experiment consisted of particles being accelerated to the required impact velocities in an air eductor where compressed air $(0.5 \mathrm{MPa})$ was allowed to expand after passing through a nozzle. The particles were introduced into the air stream individually and were impacted onto the target in random orientation. The particle velocity was determined by measuring its time of flight between a pair of photoelectric sensors located close to the target plate. The debris produced by the impact was captured with a filter on the exhaust. The target assembly was fitted with a force transducer to record the peak load experienced by the

\footnotetext{
* Corresponding author. Tel.: +31-15-278-5501; fax: +31-15-2784945.

E-mail address: R.Pitchumani@tnw.tudelft.nl (R. Pitchumani).
}

particle during impact. The initial stages of impact were recorded using an image converter camera at a speed of 50,000 frames per second. A qualitative analysis of the results was given based on the macroscopic surface features. Furthermore, they concluded that the basic mechanisms involved in fracture were qualitatively similar to those observed in quasi-static compression and indentation tests.

Salman et al. [2] performed single particle impact studies under controlled conditions against solid targets. To achieve this, a continuous air gun was employed to examine the effect of particle velocity, impact angle, particle diameter, target material, target thickness and number of impacts on the fragmentation of spherical aluminium oxide particles. The relationship between particle velocity and the number of unbroken particles was described by a two-parameter cumulative Weibull distribution. Also, increasing the particle velocity resulted in a rapid transition from zero breakage to total fragmentation for a wide range of impact angles except for an impact angle of $10^{\circ}$. Based on a similar research, Salman et al. [3] studied the failure of fertiliser granules under impact with a wide range of velocities and 
also varied the impact angle. It was observed that the failure probability for a granule at a given speed varies only slowly from normal impact $\left(90^{\circ}\right)$ down to about $50^{\circ}$, but then decreases rapidly as the angle becomes more oblique. The failure probability is also dependent on the granule size, material and impact velocity. The fragment size distributions showed that the proportion of the biggest fragments was sensitive to the impact velocity.

Vervoorn and Scarlett [4] tested impact of particles (3$\mathrm{mm}$ cylinders of pressed Alumina) against a target by repeatedly firing until a disintegrative fracture occurred. They defined disintegration a fragmentation into a number of pieces of irregular shape and also distinguished from chipping of the edges of a cylinder after impact. The process of repeated breakage events was then treated similarly to the process of grinding continuously (Vervoorn and Austin [5]) with time by defining an equivalent specific breakage factor by a first-order rate equation given by:

$w(t) W=w(0) W \exp (-S t)$

where $w(t)$ is the fraction of the total mass $W$, which is still left as original size after time of grinding $t . S$ is the specific rate of breakage. Similar method was used by Mankosa et al. [6] to study the effect of media size in stirred ball mill grinding of coal. If $t$ is considered equivalent to $N$ stages of grinding, each of which produces the same amount of breakage as $\Delta t$ of grinding time, then:

$$
\begin{aligned}
w(t) & =w(0) \exp (-S N \Delta t) \\
& =w(0) \exp (-k N)
\end{aligned}
$$

where $k$ is the equivalent to specific rate of breakage and has units of fraction per 'stage of grinding' or per 'impact action' or 'impact event'.

The definition of $k$ is not the same as that of probability of breakage per impact event because it is a differential definition which applies over a differential application of $\mathrm{d} N$,

$\frac{\mathrm{d} w(N)}{\mathrm{d} N} W=-k w(N) W$

Tests were conducted on 4 sufficiently large number of particles which enabled to obtain $w(N) / w(0)$, which is the fraction remaining unbroken after one event if $N=1$ or after $N$ events.

A number of factors were involved in the variation of the value of $k$ with the number of events for a given size of particles. These were:

(1) The distribution of strength of the particles at a standard orientation.

(2) The distribution of applied force resulting from impact.

(3) The distribution of strength of the particles due to different orientations on impact.
(4) The reduction of strength with repeated impacts due to a weakening effect.

\section{Repeated impact test}

The current research involves the development of a new impact test with the possibility of generating controlled and well-defined collisions of particles with a target. The Repeated Impact Test (RIT) is a device for characterising the impact strength of a particle. The device consists of a particle chamber in which the granules are subjected to many impacts by unidirectional movement at a predetermined frequency and amplitude. This condition is sufficient to provide reproducible damage to the particle.

The RIT test setup is aimed at simulating velocities similar to those in pneumatic conveying systems which are in the range of 5 to $25 \mathrm{~m} \mathrm{~s}^{-1}$ (Lech [7]). Furthermore, particles are expected to break or get damaged at their weakest points (Beekman [8]), and hence the impact velocity can be significantly less than those used in single particle impact experiments.

A number of prototypes were developed based on the principle of vibration with a fixed frequency and amplitude. One system involved a hydraulic piston attached to a particle chamber. The main problem with this system was that the system did not oscillate at a constant amplitude and frequency. Another prototype consisted of a vibrating system based on a resonating blade spring; see Beekman [8]. Its main disadvantages were the effect of particle mass on the resonant frequency and that the particle-particle collision was unavoidable.

The aforementioned drawbacks led to the development of the Crank Slider Type-Repeated Impact Test (CS-RIT) (Gebert et al. [9]). The main feature of this test setup is that it allows many particles to be tested at the same time and also many number of collisions. Hence, it allows the study of the mechanical behavior of particles after many controlled impacts (Table 1).

The CS-RIT can exert unidirectional movement of the particle chamber that contains a sample of particles. Contrary to previous setups, the RIT accelerates the particles towards the target and permits repeated impacts. The design of the device shown in Fig. 1 provides repeated impact of particles contained in the particle chamber in unidirectional movement and substantially only against the inner surfaces of the top and

Table 1

Part description of CS-RIT

\begin{tabular}{llcl}
\hline Part no. & Description & Part no. & Description \\
\hline 1 & linear guide shaft & 7 & rod end bearings \\
2 & linear ball splines & 8 & primary columns \\
3 & particle chamber & 9 & base plate \\
4 & sliding platform & 10 & shock absorber \\
5 & crank shaft & 11 & motor \\
6 & flywheel & 12 & secondary columns \\
\hline
\end{tabular}




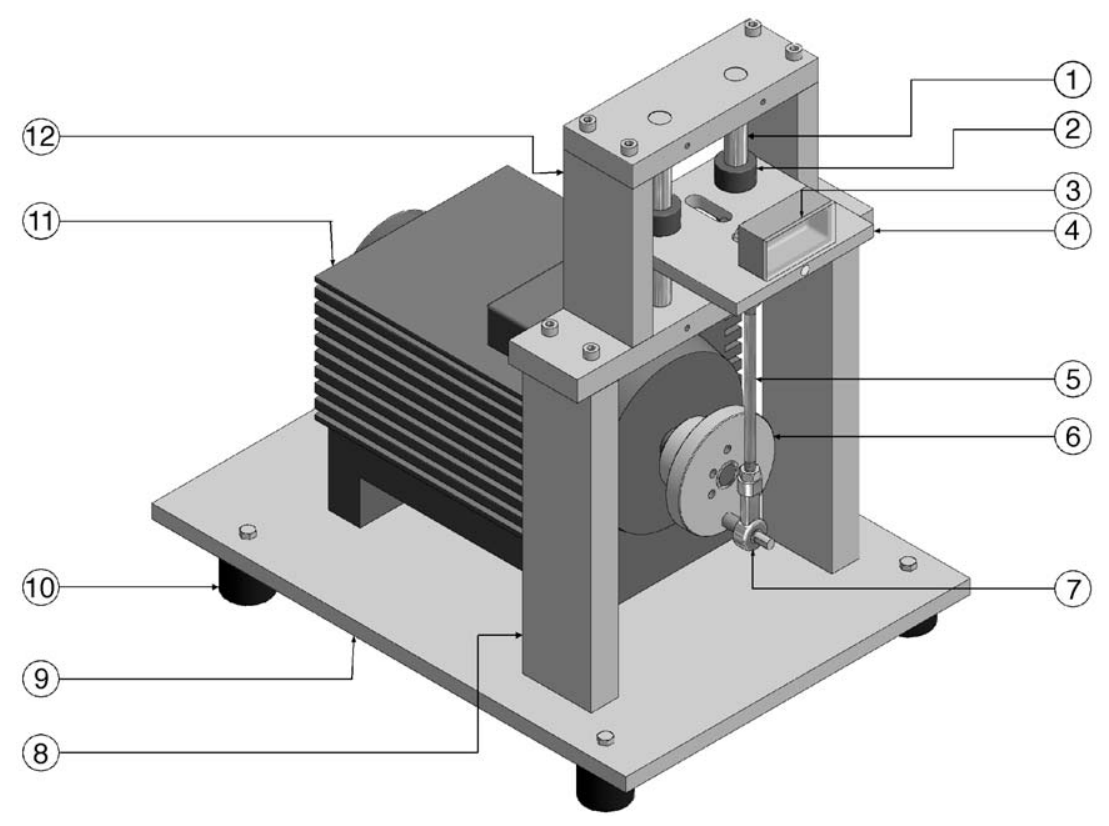

Fig. 1. Schematic drawing of the Crank Slider Type-Repeated Impact Test (CS-RIT).

bottom wall of the chamber. Furthermore, there is little or no tangential velocity component. The CS-RIT consists of a sliding platform fitted with ball bushings. The bushings could also be made of a polished metal or low friction hard plastic such as polytetrafluoroethylene. The bushings facilitate a smooth reciprocating motion of the platform along the two linear guide shafts or sliders. The guide shafts are made of very smooth stainless steel. The top and bottom ends of the guide shafts are secured with two aluminum plates which are held together by the secondary columns. The entire motion assembly rests on two primary columns which in turn are secured firmly to the base plate.

The sliding platform is attached to a flywheel with a crank shaft fitted with rod end bearings. Hence, one end of the crank shaft is attached to the sliding platform and the other to the flywheel. The crank shaft is fastened into threaded holes in the flywheel which are at an offset from the axis of rotation of the flywheel. A number of holes have been drilled in the flywheel at various radii from the axis of rotation of the flywheel. Upon rotation of the flywheel, the crank shaft is pulled downwards and pushed upwards to cause a reciprocating motion of the platform. The flywheel is powered with a three-phase motor connected to a frequency controller. The amplitude of the reciprocating platform and the container mounted on it can be varied by switching between the different threaded mounting holes. The hole nearest to the axis of rotation of the flywheel provides the lowest amplitude of movement of the platform whereas the one farthest from the axis rotation provides the highest amplitude. The motor could be any suitable drive source including an induction motor, electric motor, pneumatic pump or an internal combustion engine. An electric motor is used with a frequency controller that provides variable speed output to turn the flywheel.
The sample of particles is placed in the particle chamber where the collisions will take place and the damage is then assessed. In the selection of the particle chamber material, there were two important features: the strength of the material and its tendency to acquire charge. Since the particles collide with the walls, it is important that the walls are much stronger than the particles in question.

Initially, a particle chamber of polyethylene was used because of its light weight. This material was weak and not appropriate since the force exerted during impact is not the maximum force as most of it is absorbed by the polymer itself [8]. Polyethylene also is highly static and the particles charge during impacts. A thin dust layer (about 10 $\mu \mathrm{m})$ was formed during the experiment. This can lead to spurious results since the dust layer can absorb the impact energy.

The polyethylene box was then replaced by aluminum a stronger material. Stainless steel would have been ideal but these boxes were made from a solid block of metal and the cavity in the box had to be made by drilling through $2-\mathrm{cm}$ thick metal. At the same time, care was taken so that the walls were not thicker than $1 \mathrm{~mm}$ in order to keep the mass of the box low.

The aluminum was still weak, especially with crystals, and after a few experiments the surface of the top and bottom walls was no longer smooth. Hence, there was a need for a much stronger material. The final particle chamber has the dimensions of length $\times$ width $\times$ height $=$ $42.60 \times 18.85 \times 14.86 \mathrm{~mm}$ and is a rectangular box made of stainless steel and coated with a $100-\mu \mathrm{m}$ layer of silicon carbide, a very hard material (Knoop hardness $=15$ $\mathrm{GPa}$, Vickers hardness $=28 \mathrm{GPa}$; Cha et al. [10]), since silicon carbide is a very hard substance, ideal for strength measurements. The coating is very smooth and after 
many experiments there was no sign of damage to the coating.

The CS-RIT has the ability to control effectively the frequency of oscillation of the platform by changing the rotational speed of the flywheel. Also, because the velocity of impact of the particles is dependent on the frequency of oscillation as well as the stroke (amplitude) of the platform, the speeds can be varied with two operating parameters. A higher impact velocity can be achieved by either increasing the frequency or stroke (amplitude) or both. Increasing frequency not only increases the velocity but also reduces the time of the test. The maximum frequency which may be obtained with the device is $25 \mathrm{~Hz}$. Moreover, the desired working amplitude can be set by fastening the crank shaft into threaded holes in the flywheel which are at an offset from the axis of rotation of the flywheel.

In addition, the platform can handle larger sample sizes as the power of the motor connected to the flywheel can be very high. A large sample in a large particle chamber reduces the probability of inter-particle collisions and at the same time produces statistically correct results useful for standard tests. Since the platform is fairly large, about nine particle chambers can be mounted at the same time and the different samples can be measured. This significantly reduces the experimentation time.

The Repeated Impact Test has been developed to test the impact strength of particles. In order to test strength of any substance, it needs to be damaged. Hence, the extent of damage needs to be characterised. There are two ways to characterise damage, namely change in size of particles (Pitchumani et al. [11-16]) and shape of the particles (Pitchumani et al. [17]).

The experimental procedure depends on the method to measure damage. In this case, damage was measured by weighing the residue on a sieve. Hence, the balance used should be of high precision. It is important to have as few particles in the particle chamber as possible and at the same time a sufficiently large representative sample. This is to avoid any particle-particle collisions since the damage will be caused both due to particle-wall collisions and particleparticle collisions. Beekman [8] showed that the ratio of the area of the particle chamber and the total projected area of the particles should be more than 3 .

A sample of particles weighing between 30 and $200 \mathrm{mg}$ (depending on the particle size and density) is added to the particle chamber. The sample should contain a very narrow fraction in size of particles. The initial weight of the sample is measured accurately with a laboratory scale balance. The balance accuracy was $1 \mu \mathrm{g}$.

The content of the chamber is secured with a lid and an o-ring and fastened to each other with tape. The box is then placed on the moving platform.

The sample is subjected to impacts for residence times varied on a logarithmic time scale to ensure that conditions are well defined at both relatively low and extremely large number of particle-wall collisions. The first measurement period is $10 \mathrm{~s}$ and is followed by $20,40,80,160,320$ and $640 \mathrm{~s}$.

After each time period, the sample is taken and the debris (dust produced due to damage) is separated from the other particles by sieving through a mesh size half of the initial diameter of the particles $(425 \mu \mathrm{m})$. The weights of the mother particles are measured and then they are returned to test for further impacts. This process is continued for a total time period of $640 \mathrm{~s}$. The total time period can vary depending on the extent of damage required to be measured.

A Simple Harmonic Motion is assumed in order to calculate the number of collisions. For instance, an experiment subjected to a frequency of $25 \mathrm{~Hz}$, the particle chamber oscillates 25 times in $1 \mathrm{~s}$. It is expected that the particles collide with the top and bottom walls of the particle chamber twice in $1 \mathrm{~s}$, once with each wall. Hence, in $640 \mathrm{~s}$, there will be $640 \times 25 \times 2=32000$ collisions.

\section{Results and discussion}

In order to characterise damage by change in size, the undamaged fraction is plotted against the number of collisions. Three experiments were performed with the same amount of sample in three identical particle chambers in order to keep the conditions similar and to achieve reproducibility. The particles used in the current experiment were sodium benzoate granules. Fig. 2 shows an SEM micrograph of a sodium benzoate granule.

Fig. 3 shows the plot of the undamaged fraction versus the number of collisions corresponding to an experiment which was performed on $200 \mathrm{mg}$ of granules of size $850 \mu \mathrm{m}$ with a frequency of $25 \mathrm{~Hz}$ and amplitude of $24.55 \mathrm{~mm}$. The particles were subjected to impacts for time periods of 10 , $20,40,80,160,320,640$ s. After each time period, the sample was sieved with a $425-\mu \mathrm{m}$ sieve. The dust generated during vibration was discarded in the sieving step and the residue (or undamaged fraction) was weighed and introduced back into the particle chamber for the next time period. If $w_{0}$ is the initial mass of the sample of particles and after each time period $i$, the mass of the residue is $w_{i}$, then the fraction undamaged is defined as $w_{i} / w_{0}$.

It is observed that as the number of collisions increases there is an increase in damage since the amount of residue decreases. The attrition rate increases after the first time period. After 15000 collisions the residue is reduced to $94 \%$ of the initial mass of the sample.

One of the major advantages of the CS-RIT is that this instrument produces highly reproducible results. In all the experiments, the standard deviation of the data is less than $0.8 \%$. However, certain conditions have to be maintained to obtain such reproducible results other than the fact that the sample to be tested should be representative.

The number of particles plays an important role in the experiment. The test setup has been developed for deter- 


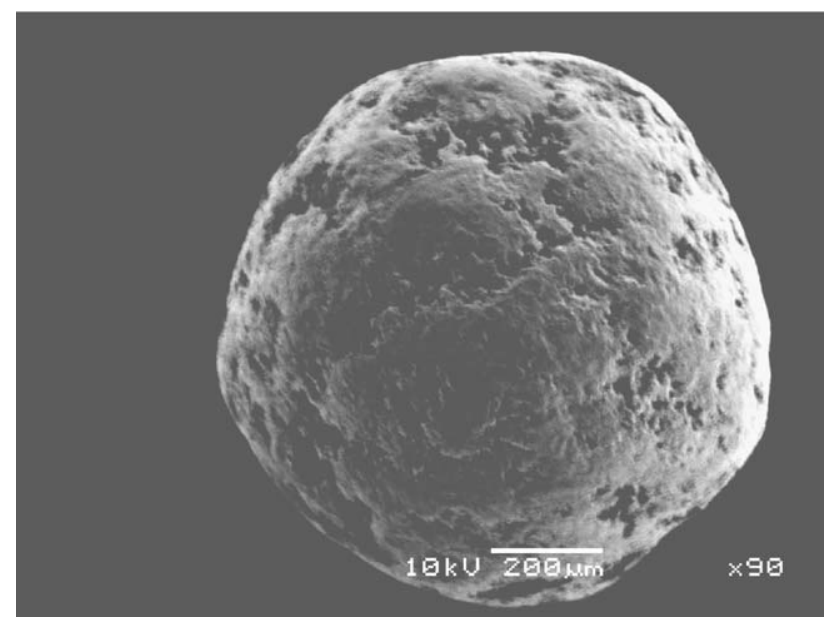

Fig. 2. SEM image corresponding to a original/untreated sodium benzoate granule of size $1180 \mu \mathrm{m}(90 \times$ magnification $)$.

mining the strength of the sample with pure particle-wall impacts, since only the particle-wall impact velocity is well defined. Particle-particle collisions cannot be determined as it is expected to vary with a wide range of values. If there are many particles in the particle chamber, then there is a high probability that particle-particle collisions will occur with high frequency. But a low number of particles results in a small sample size, thus, statistically non-representative result. Hence, constant results (reproducibility) can be obtained when the wall area is more than three or four times the total cross-sectional area of all granules. This is dependent on the particle size and the number of particles (Beekman $[8,18,19])$.

Fig. 4 shows the plot of the fraction undamaged (mass of the particles remaining on the sieve) versus the number of collisions for different number of the particles intro- duced in the particle chamber as a sample. These experiments were performed with the CS-RIT at a frequency of $25 \mathrm{~Hz}$ and an amplitude of $24.55 \mathrm{~mm}$ with sodium benzoate granules.

The experiment involved changing the number of particles in the sample in order to obtain the maximum number of particles that will avoid particle-particle interactions. The area of the wall is $42.64 \times 14.86=633.63 \mathrm{~mm}$. The average size of the particles in the sample is $d_{\mathrm{p}}=1015 \mu \mathrm{m}$. It is observed that as the number of particles increases, there is decrease in the amount of damage. The particles impact the top and bottom walls of the particle chamber normally. Hence, the total area occupied by the particles is the sum of the projected area of all the particles given by $A_{\mathrm{p}}=N_{\mathrm{p}} * \pi d_{\mathrm{p}}^{2} 4$, where $N_{\mathrm{p}}$ is the number of particles. As the number of particles increases, the area occupied by them also increases. The particles are spherical and of very narrow size fraction, so when they are packed closest to each other then the area occupied by them is the highest. This leads to very high frequency of particle-particle collisions. If there is high particle-particle collisions, then the particle-wall collisions become less. Since not all particles are subjected to particlewall impacts (which causes the highest damage), there is less breakage.

Further experiments were performed with larger particles of size $d_{\mathrm{p}}=1400 \mu \mathrm{m}$ as illustrated in Fig. 5, which is also a plot of the fraction undamaged versus the number of collisions. These experiments were performed to obtain the optimum sample size.

For a very large number of particles $N_{\mathrm{p}}=470$ and $N_{\mathrm{p}}=396$, the rate of breakage is less since the total projected area of these particles is $A_{\mathrm{p}}=722.73 \mathrm{~mm}^{2}$ and $A_{\mathrm{p}}=610.09 \mathrm{~mm}^{2}$, respectively. For lower particle numbers in the range of $N_{\mathrm{p}}=150$ to 140 , there is slightly more



Fig. 3. Plot of fraction undamaged versus number of collisions for three trials of the same sample. 




Fig. 4. Plot of fraction undamaged versus number of collisions for different amount of particles of size $1015 \mu \mathrm{m}$.

breakage as more particles are subjected to particle-wall impacts as compared to particle-particle impacts. The optimum sample is achieved for $N_{\mathrm{p}}$ less than 120 since for ranges of $N_{\mathrm{p}}$ between 130 and less, there is little difference in the amount of breakage. For $N_{\mathrm{p}}=120$, the $A_{\mathrm{p}}=184.7 \mathrm{~mm}^{2}$, and if the particles are placed in closed packing of one layer, then the total area occupied by them will be $204 \mathrm{~mm}^{2}$ and this is one-third the area of the wall. Henceforth, the area of the particles in closed packing should be one third the total area of the wall of the particle chamber.
The damage caused to the particles is due to the velocity of the impact, and the velocity in turn is dependent on the amplitude of oscillation and the frequency of oscillation. A number of experiments were performed by varying the frequency of oscillations as well as the amplitude of oscillation to observe their effect on the damage to the particles.

The experiments were performed with frequencies of 10 , 15,20 and $25 \mathrm{~Hz}$, which actually is the maximum limit of the CS-RIT, and amplitudes of 12.25, 14.5, 16.8, 20.3, 22.6 and $24.55 \mathrm{~mm}$. For each set frequency and amplitude, the experi-

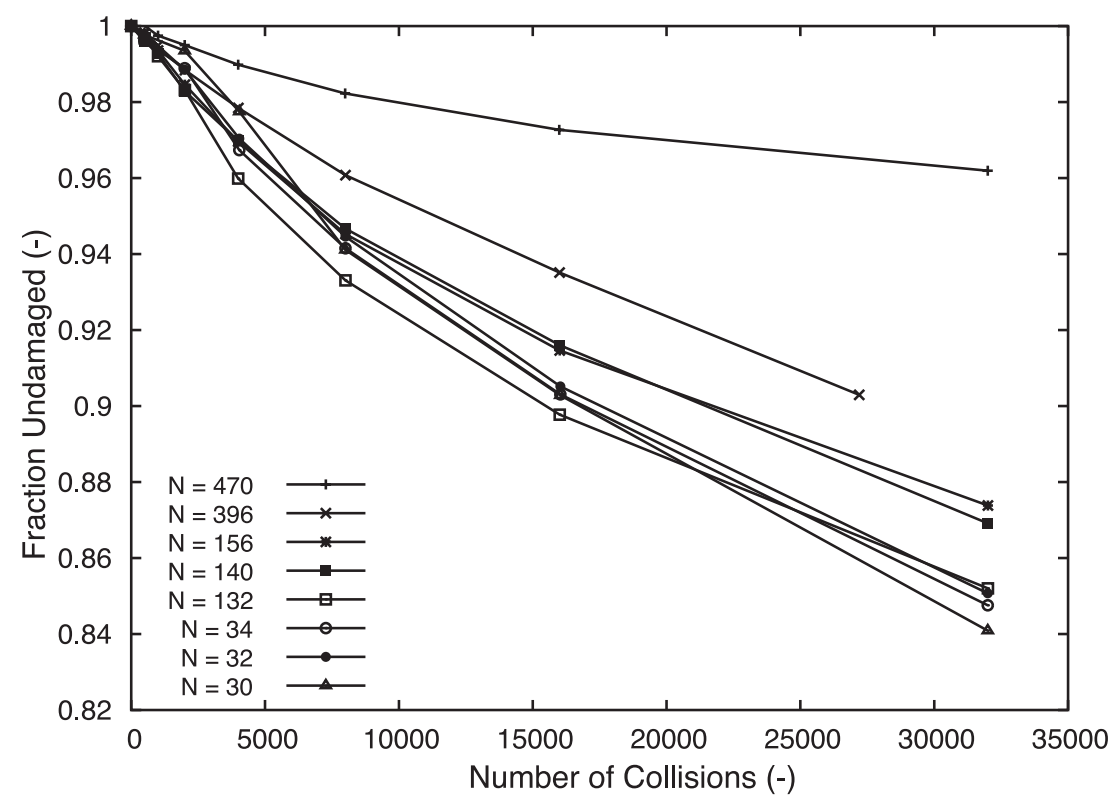

Fig. 5. Plot of fraction undamaged versus number of collisions for different amount of particles of size $1400 \mu \mathrm{m}$. 


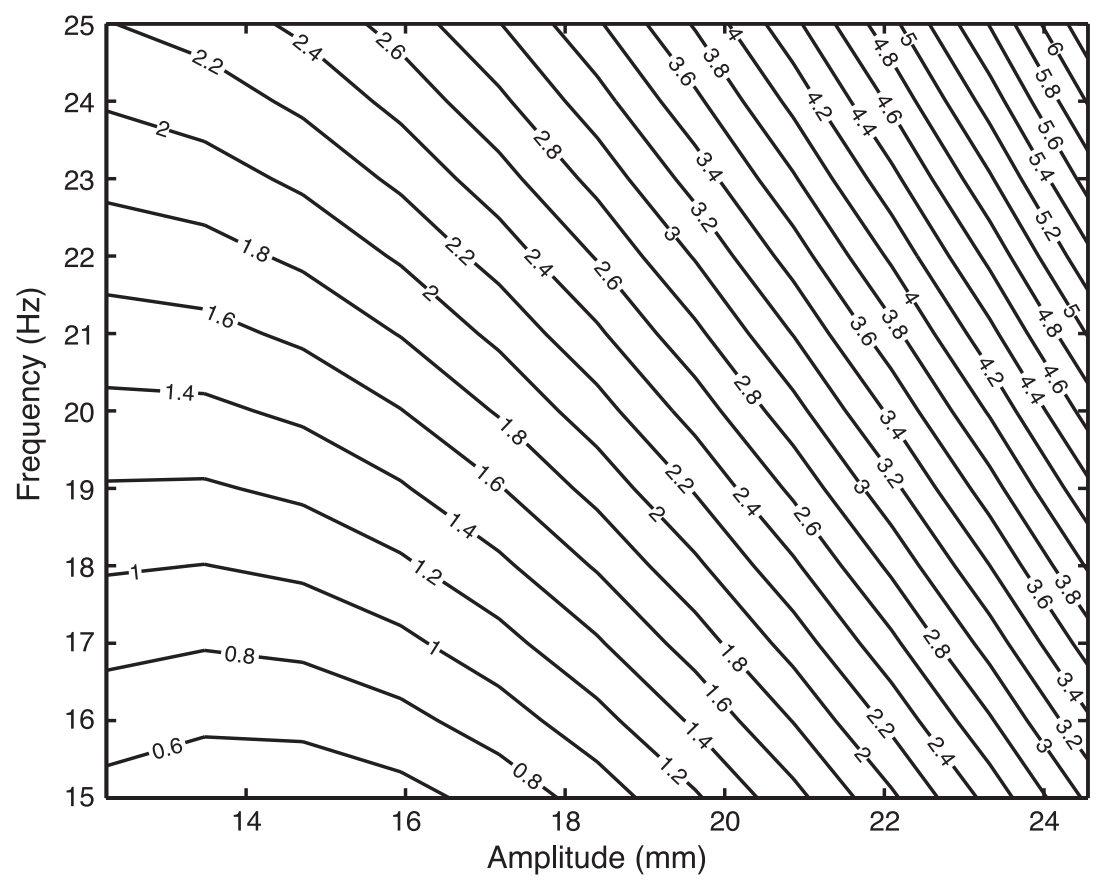

Fig. 6. Specific rate of breakage at different amplitudes and frequency for $1015-\mu \mathrm{m}$ particles.

ments were performed threefold in order to achieve concordant results. The standard deviation for all the experiments was below $0.1 \%$.

The specific rate of breakage is a measure of the rate of attrition assuming the breakage rate to be first order. Vervoorn and Austin [5] treated the process of repeated breakage events similar to that of a process of continuous grinding as explained in the introduction. But instead of $S, k$ was used, which is equivalent to specific rate of breakage and has units of fraction per 'stage of grinding' or per 'impact action' or 'impact event'.

For the current data, value of $k$ was calculated for different frequencies and amplitudes. The value for $k$ was obtained using only 1000 collisions since only in the initial stages the breakage is first order. These values of $k$ were related to amplitude $A$ and frequency, $f$, by fitting to an elliptical

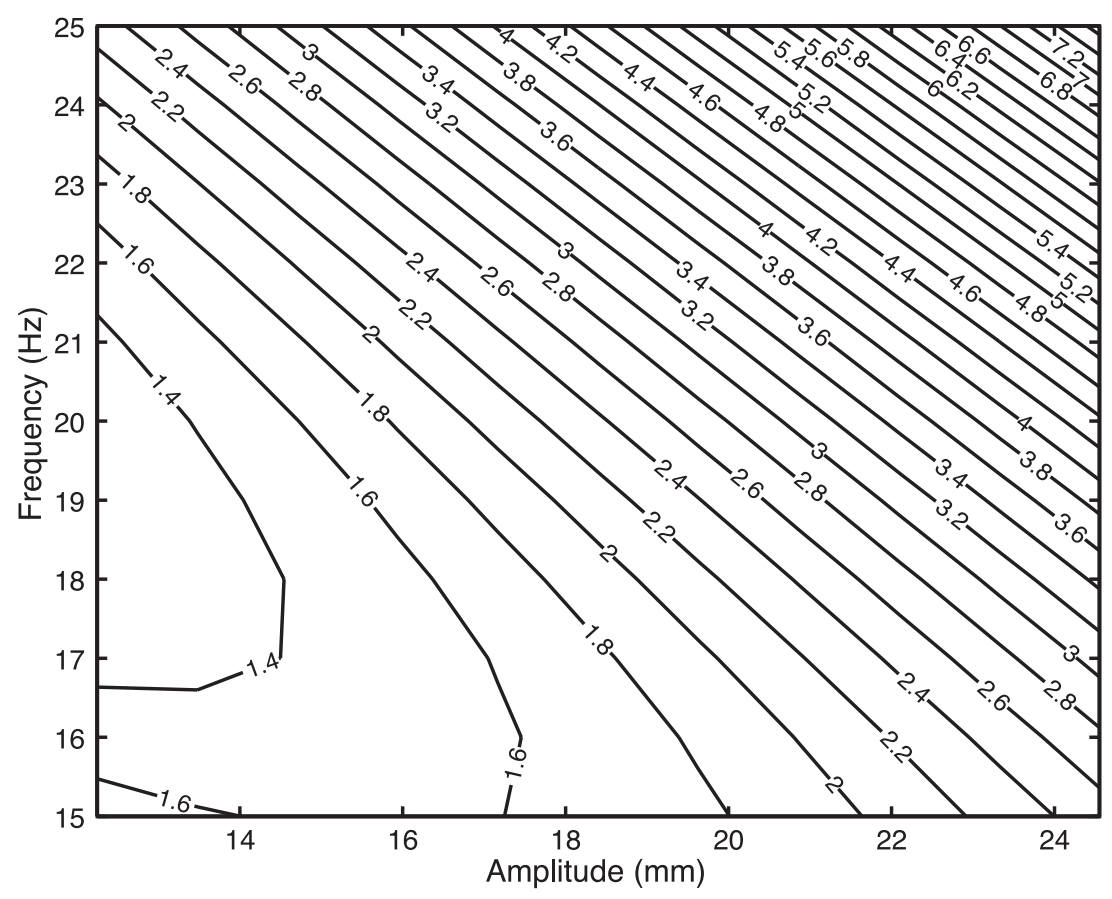

Fig. 7. Specific rate of breakage at different amplitudes and frequency for $1400-\mu \mathrm{m}$ particles. 
equation of order two. The fitting resulted in sum of squares error of less than $1 \%$. These results were then plotted as a contour plot.

Fig. 6 shows the contour plot of the variation of the specific rate of breakage with amplitude and frequency of oscillation for the $1015-\mu \mathrm{m}$ particles. It is observed from Fig. 6 that the specific rate of breakage increases with increase in amplitude and frequency. At very low amplitude and frequency, the rate is low and the initial increase in amplitude and frequency results in a gradual increase, but as both $A$ and $f$ increase, the increase in $k$ is much steeper. At lower frequencies and amplitudes, the relationship is nonlinear while at higher values it is linear.

Under ideal conditions, there are supposed to be two collisions in one oscillation. However, at low $f$ and $A$, there is less than two collisions in one oscillation. This has been confirmed with Discrete Element Modelling (Pitchumani [16]) results, which is beyond the scope of the current paper. At higher $f$ and $A$, there are exactly two collisions in one oscillation leading to a linear relationship. Similar observations can be made from Fig. 7 .

\section{Conclusions}

When a particle is subjected to impact against a target, attrition takes place where the sharp corners are broken into very fine dust and the parent particle is not fragmented. The rate of attrition is dependent on a number of factors such as material properties, particle size and shape. Attrition can be measured only by observing the damage caused to the particle. Damage is measured on the basis of change in size and shape of the particle which can be performed by either sieving or by actually using Laser diffraction or image analysis. In order to perform controlled experiments to study the effect of velocity as well as the repeated events, a new system was developed, namely the Repeated Impact Test (RIT), to replace the single particle gun experimental setup.

The RIT as a test instrument can be used to characterise the strength of particles. It has very high reproducibility with a standard deviation of less than $0.1 \%$, which is quite low compared to most other instruments available. The two operating parameters to control the RIT are frequency and amplitude of oscillation, which determine the impact velocity of the particle. A number of experiments were performed with sodium benzoate granules at different frequencies and amplitudes. The specific rate of breakage $k$ as defined by Eq. (3) [5] determines the rate of attrition during the first 1000 collisions. This value varies with different particle size and with different frequency and amplitude of oscillation. Although only one type of particle was performed in the current research, it is expected that $k$ is a function of material properties such as Young's modulus, Poisson ratio and Bulk modulus along with the impact velocity. The contour map plotted in Figs. 6 and 7 can be developed for other materials by performing similar experiments.

\section{References}

[1] K.R. Yuregir, M. Ghadiri, R. Clift, Observation of impact attrition of granular solids, Powder Technology 49 (1986) 15-22.

[2] A.D. Salman, C.A. Biggs, J. Fu, I. Angyal, M. Szabo, M.J. Hounslow, An experimental investigation of particle fragmentation using single particle impact studies, Powder Technology 128 (2002) 36-46.

[3] A.D. Salman, J. Fu, D.A. Gorham, M.J. Hounslow, Impact breakage of fertiliser granules, Powder Technology 130 (2003) 359-366.

[4] P.M.M. Vervoorn, B. Scarlett, Particle impact testing, 7th European Symposium on Comminution, Ljubljana, Yugoslavia, 1999 June, p. 195.

[5] P.M.M. Vervoorn, L.G. Austin, The analysis of repeated breakage events as an equivalent rate process, Powder Technology 63 (1990) $141-147$.

[6] M.J. Mankosa, G.T. Adel, R.H. Yoon, Effect of media size in stirred ball mill grinding of coal, Powder Technology 49 (1986) 75-82.

[7] M. Lech, Mass flow rate measurement in pneumatic conveying of solid, The Second Israel Conference for Conveying and Handling of Particulate Solids, Jerusalem, Israel, 1997 May, pp. 2.40-2.46.

[8] W.J. Beekman, Measurement of the Mechanical Strength of Granules and Agglomerates, PhD thesis, Delft University of Technology, Delft, Netherlands, 2000.

[9] M. Gebert, R. Pitchumani, W.J. Beekman, G.M.H. Meesters, B. Scarlett, Repeated impact apparatus and method for characterising granule strength. World Patent WO 02/04923 A2, World Intellectual Property Organisation, 2002 January.

[10] Y.C. Cha, G. Kim, H.J. Doerr, R.F. Bunshah, Effects of activated reactive evaporation process parameters on the microhardness of polycrystalline silicon carbide thin films, Thin Solid Films 253 (1994) 212-217.

[11] R. Pitchumani, Characterisation of strength of enzyme granules by repeated impact testing. Master's thesis, Delft University of Technology, Delft, Netherlands, 1999.

[12] R. Pitchumani, G.M.H. Meesters, B. Scarlett, Characterisation of enzyme granules by repeated impact testing, Proc. Control of Particulate Processes VI, Fraser Islands, Queensland, Australia,1999 September.

[13] R. Pitchumani, G.M.H. Meesters, B. Scarlett, Classification of breakage mechanisms of enzyme granules using repeated impact test, Proc. International Congress for Particle Technology (PARTEC), Nuremberg, Germany, 2001 March.

[14] R. Pitchumani, G.M.H. Meesters, B. Scarlett, Breakage behaviour of enzyme granules in a repeated impact test, Proc. 7th International Symposium of Agglomeration, Albi, France, 2001 July.

[15] R. Pitchumani, G.M.H. Meesters, B. Scarlett, Breakage behaviour of enzyme granules in a repeated impact test, Powder Technology 130 (2003) 421-427.

[16] R. Pitchumani, Breakage Characteristics of Particles and Granules, $\mathrm{PhD}$ thesis, Delft University of Technology, Delft, Netherlands, 2003.

[17] R. Pitchumani, N. Gupta, G.M.H. Meesters, B. Scarlett, Analysis of single particle attrition during impact experiments, Proc. World Congress on Particle Technology, Sydney Australia, 2002 July.

[18] W.J. Beekman, G.M.H. Meesters, Measurement of granule impact strength distribution in a vibrating container, Proc. World Congress on Particle Technology, vol. 3, Brighton, United Kingdom, 1998 July.

[19] W.J. Beekman, G.M.H. Meesters, B. Scarlett, T. Becker, Measurement of granule attrition and fatigue in a vibrating box, Particle and Particle Systems Characterisation 19 (2002) 5-11. 